\title{
Respuesta inmunitaria a la vacuna de la hepatitis B en pacientes hemodializados en Brasil y sus factores asociados
}

\author{
ZOE TERESINHA VICTORIA PEREIRA ${ }^{1,2, a}$, RAÚL ANDRÉS MENDOZA-SASSI ${ }^{1, \mathrm{~b}}$
}

\section{Factors associated with the immune response to hepatitis $B$ vaccine in Brazilian hemodialysis patients}

Background: Patients on chronic hemodialysis have a lower immune response to vaccination against hepatitis $B$ virus $(H B V)$ than the general population. Aim: To identify factors that may interfere with immunization against hepatitis $B$ virus $(H B V)$ in Brazilian hemodialysis patients and analyze the evolution of the level of antibodies. Patients and Methods: A retrospective longitudinal study, using records of patients on hemodialysis in the years 2000-2008. Non-responder patients, defined as a level of anti-HBs less than $10 \mathrm{IU} / \mathrm{mL}$, were identified. The effect of social and demographic factors, clinical and laboratory data on the lack of response was evaluated. Logistic regression analysis was used to assess the independent effect of each factor. The difference between initial and final anti-HBs levels (24 months), was also analyzed. Results: Fifty seven percent of patients responded adequately to vaccination. After adjustment with other variables, the only factor associated with immune response was serum ferritin. Responding patients of less than 40 years of age did not have a significant decrease in antibody titers over time. The initial anti-HBs title, influenced the final title. Fifty percent of non-responders achieved serum levels of protection after revaccination. Conclusions: The study showed that ferritin may be a marker of reduced immune response. Patients aged less than 40 years were the only ones who maintained over time their initial anti-HBs levels.

(Rev Med Chile 2012; 140: 882-888).

Key words: Hepatitis B; Immunity; Kidney failure, chronic; Renal dialysis.
L

a infección por el virus de la hepatitis B (VHB) es un grave problema de salud públide 350 millones de personas infectadas y que entre 500.000 y un millón mueren a cada año ${ }^{1}$. En 2005, el Ministerio de la Salud brasileño estimó que 15\% de la población estaría expuesta a la hepatitis B y que $1 \%$ presentaría una hepatitis $B$ crónica ${ }^{2}$.

Entre los pacientes en hemodiálisis (HD), la prevalencia es superior debido a un mayor riesgo de contagio. Estimativas del Ministerio de la Salud brasileño (2003) apuntaban para una prevalencia de $1,8 \%{ }^{3}$. Esta cifra ya fue más elevada, pero ha disminuido en función de un control riguroso de los bancos de sangre, de una menor utilización de la transfusión con el uso de la eritropoyetina y con la vacunación ${ }^{4}$. En el Brasil el programa de vacunación contra la hepatitis B se inició en 1990 y este incluye a los pacientes en HD.

Sin embargo, la respuesta entre los hemodializados crónicos es inferior que en la población general, no llegando a $60 \% \%^{5,6}$. Además el nivel de anticuerpos disminuye más rápidamente 6 . Esa respuesta inadecuada a la vacuna parece estar relacionada con el grado de insuficiencia renal ${ }^{7,8}$, y también con las alteraciones inmunitarias obser- 
vadas en los casos de uremia. Todos estos factores pueden afectar la eficacia de la vacuna incluso cuando se administra el doble de la dosis ${ }^{9,10}$. No obstante, aún existen dudas sobre cuáles son los factores que actúan en la respuesta inmunológica inadecuada en ese grupo de pacientes y sobre cuáles son los grupos dentro de los pacientes en $\mathrm{HD}$ que responden mejor a la vacunación o que mantienen por más tiempo un nivel de anticuerpos protector.

Los objetivos de este estudio fueron identificar los factores que están relacionados a la respuesta inmunitaria a la vacuna (VHB) en pacientes hemodializados e identificar que grupos presentan una respuesta inmunitaria insuficiente en un Servicio de Hemodiálisis del Brasil.

\section{Material y Método}

Fue realizado un estudio longitudinal y retrospectivo que incluyó todos los pacientes que estuvieron en hemodiálisis entre los años 2000 y 2008 en el Centro de Nefrología y Diálisis, ubicado en el Hospital Universitario Dr. Miguel Júnior Riet Correa (HU) de la Universidad de Río Grande, en el extremo sur del Estado del Río Grande del Sur, en Brasil.

Como criterio de inclusión se estableció que los pacientes debían tener una edad mínima de 18 años y estar en HD por más de un año, para obtener un mejor acompañamiento de la respuesta inmunológica después de vacunados. Los criterios de exclusión utilizados fueron: a) la vacunación anterior al período de acompañamiento; b) los pacientes con síndrome de la inmunodeficiencia adquirida; c) los pacientes ya infectados por el VHB.

Ciento dos pacientes cumplieron con los requisitos para participar del estudio. Con este tamaño de la muestra, el estudio alcanzó una potencia estadística de $80 \%$ o superior, si se considera una relación de no expuestos para expuestos al factor de riesgo de 3:1, un riesgo relativo de al menos dos, un nivel de confianza de $95 \%$ y una prevalencia de no respondedores en los no expuestos al factor de riesgo de por lo menos $35 \%$.

El estudio se desarrolló en dos etapas. La primera incluyó la totalidad de los pacientes (102) con un tiempo mínimo de permanencia en hemodiálisis de 12 meses. Al entrar al servicio, el título del anti-HBs (Anti-HBs) fue determinado para cada paciente. Los pacientes con un resultado positivo fueron considerados respondedores. Los pacientes con un resultado negativo fueron vacunados con el esquema de tres dosis de $2 \mathrm{ml}$ $(40 \mathrm{mcg})$. La vacuna utilizada fue la Engerix $B^{\circledR}$ del Laboratorio Roche. El esquema de vacunación consistió en la aplicación de una dosis de dos ml por vía intramuscular, seguida de otras dos dosis 30 y 180 días después de la primera dosis. Todos los pacientes realizaron una nueva determinación de los anticuerpos Anti-HBs después de la última dosis, siguiendo el protocolo recomendado por el Ministerio de Salud del Brasil2 ${ }^{2}$. Este procedimiento fue utilizado en todos los pacientes, y fue efectuado en diferentes momentos de acuerdo con la fecha de ingreso al programa de hemodiálisis. La caracterización como respondedor y no respondedor fue hecha en función de la respuesta a la vacunación, tomando como punto de corte $10 \mathrm{mUI} / \mathrm{ml}$.

En una segunda etapa fueron analizados sólo aquellos pacientes que pudieron ser monitoreados por lo menos durante 24 meses, incluyéndose también a los pacientes revacunados con el mismo esquema anterior de tres dosis.

En las dos etapas la periodicidad de los exámenes siguió las normas de la Secretaría Estadual de Salud, que establece: a) exámenes sanguíneos mensuales; hemograma, creatinina, urea pre y post diálisis, potasio, calcio, fósforo, transaminasa glutámica pirúvica (TGP), glucemia; b) exámenes trimestrales; medición de la saturación de la transferrina, ferritina, hierro sérico, fosfatasa alcalina y las proteínas séricas totales y sus fracciones; c) exámenes semestrales: colesterol total y las fracciones, triglicéridos y el Anti-HBs.

Los exámenes fueron realizados siempre en el mismo laboratorio, con las mismas técnicas. Para determinar el valor del Anti-HBs en cada paciente, se consideró el valor inicial, antes de la vacunación, y el final, después de la vacunación.

Como criterios para el diagnóstico de diabetes fueron seguidas las directrices de la Asociación Americana de Diabetes y adoptadas por la Asociación Brasileña, que considera como diagnóstico dos valores de glucemias en ayunas superiores a $125 \mathrm{mg} / \mathrm{dl}$. También fueron considerados diabéticos los pacientes que ya presentaban el diagnóstico de esa enfermedad antes de entrar en hemodiálisis.

Las variables independientes analizadas fueron: 
a) las variables socio-demográficas, incluyendo el sexo, la raza y la edad en años en el momento de la primera dosis de la vacuna; b) las causas de la enfermedad renal de los pacientes; c) las diversas variables bioquímicas como: los niveles medios para el período de acompañamiento de la albúmina $(\mathrm{g} / \mathrm{dl})$, ferritina $(\mathrm{ng} / \mathrm{ml})$, glucosa en sangre $(\mathrm{g} /$ $\mathrm{dl})$, calcio sérico $(\mathrm{mg} / \mathrm{dl})$, potasio $(\mathrm{mEq} / \mathrm{l})$, fósforo (mg/dl), hemoglobina (g/dl), linfocitos (células/ micro litro), el índice de remoción de urea (IRU) y la presencia de anticuerpos para la hepatitis C (anti-VHC).

El análisis fue realizado con el programa Stata 9.2. Las diferencias entre los grupos fue analizada estadísticamente mediante la prueba del $\chi^{2}$ o el test exacto de Fisher, y el test $t$ de Student o la prueba de Kruskal Wallis, según el tipo de variable y de la forma de la distribución de los datos. Posteriormente, fue realizada una regresión logística para poder ajustar los factores entre sí y obtener el efecto independiente de cada uno. Fueron calculados los odds ratios y sus intervalos de confianza de $95 \%$ (IC95). La calidad del ajuste se evaluó mediante la prueba de Hosmer-Lemeshow. En todas las pruebas se utilizó como criterio de significancia estadística un $\mathrm{p} \leq 0,05$.

El estudio fue aprobado por el Comité de Ética en Pesquisa para seres humanos de la Universidad Federal de Río Grande.

\section{Resultados}

\section{Análisis de la muestra}

De los 116 pacientes que estuvieron en HD durante el período del estudio, ciento dos cumplieron los criterios de inclusión-exclusión y fueron reclutados. En las Tablas 1 y 2 se describe la distribución de las variables sociodemográficas, clínicas y bioquímicas. La edad media fue 55,3 (DE 21,2), con límites de 18 a 88 años. La mayor parte eran hombres (57\%), blancos $(77 \%)$ y con 60 años o más (41\%). La hepatitis C, tuvo una prevalencia de $25,5 \%$. La causa de insuficiencia renal más frecuente fue la Diabetes Mellitus (38,4\%). Los valores bioquímicos estuvieron dentro de la normalidad con excepción del fósforo, el potasio y la urea pre diálisis que estaban elevados. Después de completar el protocolo de vacunación, en 58 pacientes (56,9\%; IC95 47,1- 66,6) se observó una seroconversión, con valores de anti-HBs iguales o superiores a $10 \mathrm{mUI} / \mathrm{ml}$.
Diferencias entre las características de los respondedores y no respondedores

En la Tabla 3 están descritas las diferencias entre los respondedores y los no respondedores. Las únicas diferencias significativas encontradas entre los dos grupos fueron la ferritina $(\mathrm{p}=0,01)$ y la glucosa $(\mathrm{p}=0,05)$.

Al realizar el ajuste de los datos mediante la regresión logística, la única variable que permaneció significativa en el modelo fue la ferritina, con una RO de 0,998, (IC95 0,996-0,999). De forma que el aumento de la concentración de esa sustancia determinó una disminución en la chance del paciente a ser respondedor.

\section{Análisis de los cambios en los títulos}

de anti-HBs durante 24 meses

De los 102 pacientes iniciales, 59 continuaron en diálisis por un período igual o superior a 24

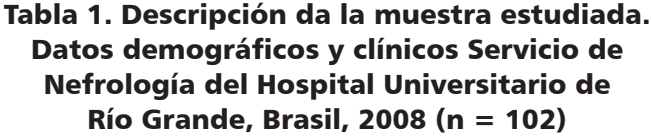

\begin{tabular}{|lrc|}
\hline Variable & $\%$ & $\mathbf{n}$ \\
\hline Edad en la vacunación (años completos) & & \\
$<40$ años & 18,63 & 19 \\
$\geq 40$ años & 81,37 & 83 \\
\hline Color de la piel & & \\
Blanca & 77,45 & 79 \\
Negra & 15,69 & 16 \\
Parda & 6,86 & 07 \\
Sexo & & \\
Varón & 56,86 & 58 \\
Mujer & 43,14 & 44 \\
\hline Etiología de la enfermedad renal & & \\
Nefropatía diabética & 38,24 & 39 \\
Glomerulopatía crónica & 23,53 & 24 \\
Nefropatía hipertensiva & 14,71 & 15 \\
Nefropatía Intersticial crónica & 10,78 & 11 \\
Otras nefropatías o indeterminadas & 12,74 & 13 \\
\hline Serología de la hepatitis C positiva & 25,49 & 26 \\
\hline Diabetes Mellitus & 40,20 & 41 \\
\hline Respondedores a la vacuna & 56,86 & 58 \\
(> 10 mUl/ml) & & \\
\hline No respondedores a la vacuna & 43,14 & 44 \\
(<= 10 mUl/ml) & & \\
\hline
\end{tabular}


Tabla 2. Descripción da la muestra estudiada. Análisis de laboratorio. Servicio de Nefrología del Hospital Universitario de Río Grande, Brasil, 2008 (n = 102)

\begin{tabular}{|lrrcc|}
\hline Parámetros bioquímicos & Media & \multicolumn{1}{c}{ DE } & Rango & Valores normales \\
\hline Albúmina $(\mathrm{g} / \mathrm{dl})$ & 3,60 & 0,38 & $2-4,35$ & 3,5 a $5,00 \mathrm{~g} / \mathrm{dl}$ \\
\hline Ferritina $(\mathrm{ng} / \mathrm{ml})$ & 315,28 & 365,51 & $12-2.242$ & 11,0 a $336,2 \mathrm{ng} / \mathrm{ml}$ \\
\hline Urea pre-diálisis $(\mathrm{mg} / \mathrm{dl})$ & 133,75 & 30,68 & $64-244,33$ & 15 a $40 \mathrm{mg} / \mathrm{dl}$ \\
\hline Índice de remoción de urea (\%) & 65,69 & 7,13 & $50-99,38$ & Acima de $65 \%$ \\
\hline Calcio $(\mathrm{mg} / \mathrm{dl})$ & 8,93 & 0,58 & $7,33-10,3$ & 8,8 a $11,0 \mathrm{mg} / \mathrm{dl}$ \\
\hline Fósforo $(\mathrm{mEq} / \mathrm{l})$ & 5,75 & 1,42 & $3,2-10,15$ & 2,5 a $4,8 \mathrm{mg} / \mathrm{dl}$ \\
\hline Potasio $(\mathrm{mEq} / \mathrm{l})$ & 5,12 & 0,60 & $3,7-6,83$ & 3 a $5 \mathrm{mEq} / \mathrm{l}$ \\
\hline Glucemia $(\mathrm{mg} / \mathrm{dl})$ & 108,00 & 46,86 & $58-410$ & 70 a $110 \mathrm{mg} / \mathrm{dl}$ \\
\hline Hemoglobina $(\mathrm{g} \%)$ & 8,93 & 1,16 & $6,9-13,2$ & 11 a $16 \mathrm{~g} \%$ \\
\hline Linfocitos $(\mathrm{cels} / \mu \mathrm{l})$ & $2.085,82$ & 773,66 & $623-4.628$ & 1.000 a $4.500 / \mathrm{ul}$ \\
\hline
\end{tabular}

meses y pudieron ser acompañados durante todo ese período. En ese grupo fueron determinados los valores iniciales y finales de los anticuerpos. El promedio de anti-HBs inicial fue 359,8 (DE 373,5) y el final fue de 237,4 (DE330,5) ( $\mathrm{p}=0,001)$.

De los 59 pacientes en seguimiento, 41 (69,5\%) eran respondedores.

Los títulos de anti-HBs iniciales no fueron significativamente diferentes entre las categorías varones y mujeres $(\mathrm{p}=0,4)$, pacientes menores de 40 años y con 40 años o más $(\mathrm{p}=0,2)$, y pacientes con o sin diabetes $(\mathrm{p}=0,1)$. Tampoco hubo una diferencia significativa entre las distintas categorías de esos grupos en los títulos finales (Tabla 4).

Cuando se analizaron las diferencias entre los títulos iniciales y finales de anti-HBs, todas las categorías de las variables estudiadas mostraron diferencias significativas. La única excepción fue la categoría de pacientes menores de 40 años, que mantuvieron títulos estables durante los 24 meses (Tabla 5). No se encontraron diferencias significativas entre las medias iniciales de las diferentes categorías. Lo mismo ocurrió con las medias finales.

Al correlacionar los títulos iniciales de antiHBs con los títulos finales, se observó una correlación fuerte, con un $\mathrm{R}=0,8$ y $\mathrm{p}=0,008$.

La proporción de pacientes con títulos iniciales elevados de anticuerpos aumentó de acuerdo al aumento del título inicial del paciente $(\mathrm{p}=0,0001)$.

La totalidad de los 11 pacientes que alcanzaron un nivel superior a $500 \mathrm{mUI} / \mathrm{ml}$ en la primera medida, se mantuvieron en esa categoría dos años después de la vacunación (Tabla 6).

Entre los 18 pacientes no respondedores que fueron revacunados, nueve (50\%) tuvieron una seroconversión. Por lo tanto, si se toma en cuenta la vacunación inicial $(56,9 \%)$ y la revacunación, se obtuvo una respuesta total de $65,7 \%$.

\section{Discusión}

Este estudio tuvo como objetivo identificar los posibles factores asociados a la seroconversión después de la vacunación contra la hepatitis B en una muestra de pacientes en HD y estudiar la evolución del título del anti-HBs en los 24 meses siguientes.

La tasa de seroconversión encontrada (56,9\%), fue muy parecida a la encontrada en los estudios previos: español $(54,4 \%)^{11}$ y norteamericano $(50 \%)^{12}$. No fue posible establecer una asociación entre el estado de respondedor y los factores socio demográficos o clínicos analizados. Sin embargo, en otros estudios se ha encontrado una respuesta inadecuada a la vacunación en pacientes diabéti$\cos ^{13} \mathrm{o}$ con hepatitis $\mathrm{C}^{14}$.

La media de anticuerpos a lo largo del tiempo se mantuvo entre los pacientes con una edad inferior a 40 años, como fue mostrado en otro estudio ${ }^{15}$ y este hallazgo puede estar relacionado a los cambios fisiológicos e inclusive inmunológicos, que acompañan al envejecimiento.

Con respecto a los parámetros bioquímicos, solamente la ferritina sérica mantuvo una aso- 
Tabla 3. Comparación de los datos demográficos, sociales y clínicos entre los pacientes respondedores y no respondedores. Servicio de Nefrología del Hospital Universitario de Río Grande, Brasil, 2008 (n = 102)

\begin{tabular}{|c|c|c|c|c|c|}
\hline \multirow[t]{2}{*}{ Variables } & \multicolumn{2}{|c|}{ Respondedores (58) } & \multicolumn{3}{|c|}{ No Respondedores (44) } \\
\hline & $\%$ (n) & $\mathbf{n}$ & $\%(n)$ & $\mathbf{n}$ & $\mathbf{p}$ \\
\hline \multicolumn{6}{|c|}{ Edad en la vacunación (años completos) } \\
\hline$<40$ años & 63,16 & 12 & 36,84 & 7 & \multirow{2}{*}{0,5} \\
\hline$\geq 40$ años & 55,42 & 46 & 44,58 & 37 & \\
\hline \multicolumn{6}{|l|}{ Color de la piel } \\
\hline Blanca & 57,69 & 45 & 42,31 & 33 & \multirow{2}{*}{0,8} \\
\hline No blanca & 54,17 & 13 & 45,83 & 11 & \\
\hline \multicolumn{6}{|l|}{ Sexo } \\
\hline Varón & 62,07 & 36 & 37,93 & 22 & \multirow{2}{*}{0,2} \\
\hline Mujer & 50,00 & 22 & 50,00 & 22 & \\
\hline \multicolumn{6}{|l|}{ Etiología de la IRC } \\
\hline Diabéticos & 56,41 & 22 & 43,59 & 17 & \multirow[b]{2}{*}{0,9} \\
\hline No diabéticos & 57,14 & 36 & 42,86 & 27 & \\
\hline \multicolumn{6}{|l|}{ Hepatitis C } \\
\hline Positivo & 69,23 & 18 & 30,77 & 8 & \multirow{2}{*}{0,1} \\
\hline Negativo & 52,63 & 40 & 47,37 & 36 & \\
\hline Diabetes Mellitus & 48,78 & 20 & 51,22 & 21 & 0,2 \\
\hline Parámetros bioquímicos & Media & DE & Media & DE & $\mathbf{P}$ \\
\hline Albúmina $(\mathrm{g} / \mathrm{dl})$ & 3,60 & 0,42 & 3,61 & 0,33 & 0,4 \\
\hline Ferritina (nanog/ml) & 243,62 & 230,37 & 415,62 & 482,59 & 0,01 \\
\hline Urea pre-diálisis (mg/dl) & 137,94 & 32,12 & 127,96 & 27,91 & 0,9 \\
\hline Índice de remoción de urea (\%) & 65,39 & 8,40 & 66,10 & 4,94 & 0,3 \\
\hline Calcio $(\mathrm{mg} / \mathrm{dl})$ & 8,98 & 0,57 & 8,86 & 0,60 & 0,8 \\
\hline Fósforo (meq/l) & 5,80 & 1,36 & 5,69 & 1,51 & 0,6 \\
\hline Potasio (meq/l) & 5,19 & 0,56 & 5,04 & 0,65 & 0,9 \\
\hline Glucemia (mg/dl) & 101,39 & 36,41 & 116,69 & 57,16 & 0,05 \\
\hline Hemoglobina (g\%) & 9,11 & 1,18 & 8,66 & 1,08 & 0,9 \\
\hline Linfocitos (cels/ $\mu \mathrm{l})$ & $2.115,26$ & 898,86 & $2.046,57$ & 574,00 & 0,7 \\
\hline
\end{tabular}

Tabla 4. Seroconversión y títulos medios de anti-HBs $(\mathrm{mUl} / \mathrm{ml})$ después de tres dosis de la vacuna contra hepatitis $B$, para la edad, el sexo y la diabetes. Servicio de Nefrología del Hospital Universitario de Río Grande, Brasil $(n=41)$

\begin{tabular}{|llc|}
\hline \multirow{3}{*}{ Sexo } & & Títulos medios (DE) \\
& Varón & 214,31 (DE 334,45) \\
& Mujer & $149,28(\mathrm{DE} 263,84)$ \\
\multirow{3}{*}{ Edad } & $<40$ años & $290,32(\mathrm{DE} 430,67)$ \\
& $\geq 40$ años & 163,96 (DE 271,79) \\
\multirow{2}{*}{ Diabetes } & Sí & 120,65 (DE 233,58) \\
& No & 230,36 (DE 350,83) \\
\hline
\end{tabular}

$p>0,05$. ciación inversa con el estado de respondedor, en el modelo multivariado. Esa asociación ya fue descrita anteriormente entre pacientes en hemodiálisis vacunados contra la gripe ${ }^{17}$, pero no hubo hasta ahora ningún relato de esa relación con la vacuna contra la hepatitis B. Una explicación para este hallazgo es que la ferritina tendría un efecto supresor de la respuesta inmune, aunque esa función aún no está bien establecida. Otros estudios muestran que los niveles de ferritina se modifican en la respuesta inmunológica ${ }^{17,18}$.

Otra hipótesis posible es que la ferritina es en realidad un marcador de la inflamación en la IRC $^{19}$. Estudios recientes indican que la hepcidina 
Tabla 5. Distribución de títulos de anti HBs iniciales y finales según el sexo, la edad y la diabetes en pacientes hemodializados en seguimiento de 24 meses post vacunación Anti VHB. Servicio de Nefrología del Hospital Universitario de Río Grande, Brasil $(n=41)$

\begin{tabular}{|llccc|}
\hline Variable & & Anti-Hbs inicial & Anti-Hbs final & p \\
\multirow{2}{*}{ Sexo } & Varón & 352,19 & 190,02 & 0,004 \\
\multirow{2}{*}{ Edad } & Mujer & 373,03 & 319,64 & 0,04 \\
& $<40$ años & 416,67 & 389,94 & 0,15 \\
\multirow{2}{*}{ Diabetes } & $\geq 40$ años & 346,03 & 200,47 & 0,001 \\
& Sí & 296,47 & 189,77 & 0,005 \\
\hline
\end{tabular}

Tabla 6. Distribución de rangos de anti HBS según la respuesta inicial y final en pacientes hemodializados en seguimiento de 24 meses post vacunación Anti VHB. Servicio de Nefrología del Hospital Universitario de Río Grande, Brasil 2008 ( $n=41)$

\begin{tabular}{|lcccc|}
\hline & \multicolumn{5}{c|}{ Respuesta a los $\mathbf{2 4}$ meses } \\
Respuesta Inicial & $<\mathbf{1 0}$ & $\mathbf{1 0 - 1 0 0}$ & $\mathbf{1 0 1}<\mathbf{5 0 0}$ & $>\mathbf{5 0 0}$ \\
$10-100$ & $4(33,3 \%)$ & $8(66,7 \%)$ & 0 & 0 \\
$101-500$ & $4(22,2 \%)$ & $5(27,8 \%)$ & $9(50 \%)$ & 0 \\
$>500$ & 0 & 0 & 0 & $11(100 \%)$ \\
\hline
\end{tabular}

Test exacto de Fisher $p=0,0001$.

iniciaría ese aumento de la ferritina en el proceso inflamatorio. Esta sustancia es un péptido producido en el hígado, responsable por la regulación sistémica del metabolismo del hierro y que cuando existe un proceso inflamatorio, infeccioso o neoplásico provoca el aumento de este ión en los macrófagos y en otras células ${ }^{20,21}$. Sin embargo, aún no ha sido determinado completamente el papel de la ferritina y su relación con la respuesta inmunológica en la vacunación contra el VHB en pacientes hemodializados. Lo que nosotros hemos puesto en evidencia es que su aumento está asociado a una disminución de la respuesta inmunológica en esos pacientes, ya sea porque es un marcador de otra sustancia o por algún otro mecanismo no conocido.

Nuestro estudio observó también que la persistencia de anticuerpos está relacionada al nivel de títulos obtenido inicialmente y que niveles elevados garantizan una mayor persistencia de la respuesta. Resultados similares fueron obtenidos en el estudio de Pin et $\mathrm{al}^{11}$.

En nuestro estudio, la tasa de seroconversión entre los no respondedores, en que se utilizó la vía intramuscular, como se recomienda, fue de $50 \%$, valor muy similar al encontrado en el grupo que utilizó la misma vía (48\%), en un estudio en el que se compararon las diferentes vías de administración ${ }^{22}$.

Entre las limitaciones del presente estudio debemos tener en cuenta que no fue posible evaluar la transferencia pasiva de anticuerpos a través de transfusiones previas. No hay pruebas para descartar esta posibilidad, pero el número de pacientes que podrían tener niveles elevados de anticuerpos debido a la transferencia pasiva es bajo.

Otra limitación es que no fue medida directamente la tasa de adhesión completa al calendario de vacunación, dado que el procedimiento no es realizado en el servicio de Hemodiálisis y sí en una unidad específica de la secretaria de Salud del municipio. Esta posible limitación puede ser minimizada si se considera que todos los pacientes participantes del estudio tenían registrado en un carné de vacunación las dosis recibidas y los datos eran inmediatamente anotados en el registro electrónico a partir del cuál se recogieron los datos. Si esa información faltase el paciente era alertado sobre la situación y la familia también era comunicada. 
Para concluir, el presente estudio encontró una tasa de no respondedores elevada y similar a otros estudios realizados. Entre los aspectos a destacar, se consiguió demostrar una relación inversa entre la ferritina y la respuesta inmunológica, pero el papel de esa sustancia en el proceso inmunológico que ocurre en la vacunación de los pacientes hemodializados es aún desconocido. Existe la sospecha que pueda ser un marcador del proceso inflamatorio. Nuevos estudios deberán aclarar si puede ser utilizada como un factor pronóstico de los pacientes no respondedores o si es una de las causas de la respuesta inmunológica insatisfactoria.

Otro aspecto que se concluye de los resultados del estudio es que la persistencia de los niveles de anticuerpos depende de los niveles iniciales obtenidos.

\section{Referencias}

1. Maynard JE. Hepatitis B: Global importance and need for control. Vaccine 1990; 8 (Suppl): S18-20.

2. Ministerio da Saúde-Brasil. Hepatites virais: o Brasil esta atento. Brasilia (DF): Ministério da Saúde, Secretaria de Políticas de Saúde, Programa Nacional de Hepatites Virais; 2003.

3. Ministerio da Saúde - Brasil. Guia de Vigilância Epidemiológica. Brasilia (DF): Ministério da Saúde; 2005.

4. Molino C, Fabbian F, Cozzolino M, C L. The management of viral hepatitis in CHD patients: an unresolved problem. Int J Artif Organs 2008; 31 (8): 683-96.

5. Buti M, Viladomiu L, Jardi R, Olmos A, Rodrigues JA, Bartolomé J, et al. Long-term immunogenicity and efficay of hepatitis B vaccine in hemodialysis patients. Am J Neprol 1992; 12 (3): 144-7.

6. Tang S, Lai KN. Chronic viral hepatitis in hemodialysis patients Hemodial Int 2005; 9 (2): 169-79.

7. Dinits-Pensy M, Forrest GN, Cross AS, Hise MK. The use of vaccines in adult patients with renal disease. Am J Kidney Dis 2005; 46 (6): 997-1011.

8. Kausz A, Pahari D. The value of vaccination in chronic kidney disease. Semin Dial 2004; 17 (1): 9-11.

9. Bricks LF. Indicação de vacinas e imunoglobulinas em indivíduos que apresentam comprometimento da imunidade. Rev Saude Publica 1998; 32 (3): 281-94.

10. Vaisbich MH, Furusawa EA, Fujimura MD, Diogo CL, Oliveira LC, Okay Y, et al. Vacina contra hepatite B em crianças com insuficiência renal com tratamento con- servador. J Bras Nefrol 1998; 20 (2): 138-43.

11. Pin M, Compte MT, Angelet P, Gallego C, Gutiérrez C, Martínez Vea A. Evaluación a largo plazo de la respuesta imunológica a la vacuna de la hepatitis B en 136 pacientes en hemodiálisis. Nefrología 2009; 29 (5): 415-20.

12. Stevens CE, Alter HJ, Taylor PE, Zang EA, Harley EJ, Szmuness W. Hepatitis B vaccine in patients receiving hemodialysis. Immungenicity and efficacy. N Engl J Med 1984; 311 (8): 496-501.

13. Fiçicio lu C, Mikla S, Midilli K, Aydin A, Cam H, S. E. Reduced immune response to hepatitis $\mathrm{B}$ vaccine in children with insulin dependent diabetes. Acta Paediatr Jpn 1995; 37 (6): 687-90.

14. Navarro JF, Teruel JL, Mateos M, J. O. Hepatitis C virus infection decreases the effective antibody response to hepatitis B vaccine in hemodialysis patients. Clin Nephrol 1994; 41 (2): 113-6.

15. Peces R, de la Torre M, Alcázar R, JM. U. Prospective analysis of the factors influencing the antibody response to hepatitis B vaccine in hemodialysis patients. Am J Kidney Dis 1997; 29 (2): 239-45.

16. Ibrahim S, el-Din S, I. B. Antibody level after hepatitis-B vaccination in hemodialysis patients: impact of dialysis adequacy, chronic inflammation, local endemicity and nutritional status. J Natl Med Assoc 2006; 98 (12): 19537.

17. Eiselt J, Kielberger L, Sedlácková T, Racek J, P P. High ferritin, but not hepcidin, is associated with a poor immune response to an influenza vaccine in hemodialysis patients. Nephron Clin Pract 2010; 115 (2): 147-53.

18. Matzner Y, Hershko C, Polliack A, Konijn AM, G I. Suppressive effect of ferritin on in vitro lymphocyte function. Br J Haematol 1979; 42 (3): 345-53.

19. Kalantar-Zadeh K. Inflammatory marker mania in chronic kidney disease: pentraxins at the crossroad of universal soldiers of inflammation. Clin J Am Soc Nephrol 2007; 2 (5): 889-97.

20. Ganz T. Hepcidin, a key regulator of iron metabolism and mediator of anemia of inflammation. Blood 2003; 102 (3) :783-8.

21. Vyoral D, Petrák J. Hepcidin: a direct link between iron metabolism and immunity. Int J Biochem Cell Biol 2008; 37 (9): 1768-73.

22. Fabrizi F, Andrulli S, Bacchini G, Corti M, Locatelli F. Intradermal versus intramuscular hepatitis B revaccination in non-responsive chronic dialysis patients: a prospective randomized study with cost-effectiveness evaluation. Nephrol Dial Transplant 1997; 12 (6): 1204 11. 